\title{
Estratégias de ensino para a aprendizagem sobre sepse
}

Teaching strategies for learning about sepsis

Estrategias de enseñanza para aprender sobre sepsis

\section{Douglas de Oliveira Silva ${ }^{I}$, Izabela Katiucia Tomaz da Silva ${ }^{I I}$, Laís Silva Palmieri ${ }^{I I I}$ Fabiana Cristina Pires ${ }^{\mathrm{IV}}$, Juliana da Silva Garcia Nascimentov}

\begin{abstract}
Resumo: Objetivo: verificar a efetividade da articulação de métodos tradicionais e ativos de ensino para desenvolver conhecimento de estudantes de enfermagem sobre sepse. Método: estudo quase experimental, transversal, do tipo antes e depois, realizado com 27 estudantes de enfermagem por meio de um workshop sobre sepse. Houve aplicação de 5 estratégias de ensino: palestra expositiva, paródia sobre sepse, debate entre equipes, jogo da memória e estudo de caso, seguidas de teste cognitivo. Após 3 meses, ocorreu nova aplicação do teste cognitivo. Resultados: constatou-se aumento da média de acertos no pós-teste imediato com significância estatística $(\mathrm{p}<0,001)$. Três meses após, a média de acertos foi próxima ao teste anteriormente realizado, porém, não houve relevância significativa ( $>0,05)$ para retenção do conhecimento. Conclusão: a articulação de métodos tradicionais e ativos de aprendizagem provocam desenvolvimento de conhecimento sobre a sepse.

Descritores: Educação em enfermagem; Conhecimento; Sepse; Metodologias ativas; Estratégias de ensino e aprendizagem
\end{abstract}

Abstract: Objective: To verify the effectiveness of the articulation of traditional and active teaching methods to develop nursing students' knowledge about sepsis. Method: quasi-experimental, cross-sectional, before and after study conducted with 27 nursing students through a sepsis workshop. Five teaching strategies were applied: expository lecture, sepsis parody, team debate, memory game and case study, followed by cognitive testing. After 3 months, there was a new application of the cognitive test. Results: there was an increase in the average of right answers in the immediate post-test with statistical significance $(p<0.001)$. Three months later, the average of correct answers was close to the previous test, but there was no significant relevance ( $p>0.05$ ) for knowledge

\footnotetext{
I Enfermeiro, Universidade de Uberaba (UNIUBE), Uberaba, Minas Gerais, Brasil. E-mail: douglasgrafitenp@gmail.com. ORCID: https://orcid.org/0000-0003-4669-7598

II Enfermeira, Universidade de Uberaba (UNIUBE), Uberaba, Minas Gerais, Brasil. E-mail: iza_tomaz@hotmail.com. ORCID: https:/orcid.org/00000003-3387-3513

III Enfermeira, Universidade de Uberaba (UNIUBE), Uberaba, Minas Gerais, Brasil. E-mail: lais.palmieri.silva@hotmail.com. ORCID: https://orcid.org/0000-0002-7951-2804

IV Enfermeira, Especialista em Urgência e Emergência, Universidade Federal do Triângulo Mineiro, Uberaba, Minas Gerais, Brasil. E-mail: enfermagem.pires@gmail.com. ORCID: https://orcid.org/0000-0002-8524-1449

v Enfermeira, Mestre em Enfermagem, Universidade de São Paulo, Escola de Enfermagem de Ribeirão Preto (EERP-USP), Ribeirão Preto, São Paulo. E-mail: mestradounesp28@yahoo.com.br. ORCID: https://orcid.org/0000-0003-1118-2738
} 
retention. Conclusion: the articulation of traditional and active learning methods lead to the development of knowledge about sepsis.

Descriptors: Nursing education; Knowledge; Sepsis; Active methodologies; Teaching and learning strategies

Resumen: Objetivo: Verificar la efectividad de la articulación de métodos de enseñanza tradicionales y activos para desarrollar el conocimiento de los estudiantes de enfermería sobre la sepsis. Método: estudio cuasi-experimental, transversal, antes y después realizado con 27 estudiantes de enfermería a través de un taller de sepsis. Se aplicaron cinco estrategias de enseñanza: conferencia expositiva, parodia de sepsis, debate en equipo, juego de memoria y estudio de caso, seguido de pruebas cognitivas. Después de 3 meses, hubo una nueva aplicación de la prueba. Resultados: hubo un aumento en el promedio de respuestas correctas en la prueba posterior inmediata con significancia estadística $(\mathrm{p}<0.001)$. Tres meses después, el promedio de respuestas correctas estuvo cerca de la prueba anterior, pero no hubo relevancia significativa ( $>$ 0.05) para la retención del conocimiento. Conclusión: la articulación de los métodos de aprendizaje tradicionales y activos conducen al desarrollo del conocimiento sobre la sepsis.

Descriptores: educación en enfermería; Conocimiento; Sepsis; Metodologías activas; Estrategias de enseñanza y aprendizaje

\section{Introdução}

A educação em saúde ainda é, na maioria das vezes, baseada em um modelo fragmentado do saber, centrada no professor, desconsiderando as necessidades de atuação na prática e fundamentadas geralmente, em métodos tradicionais de ensino e aprendizagem na saúde. ${ }^{1}$

A preferência por mecanismos exclusivamente tradicionais para promover o aprendizado em saúde como as palestras frequentemente adotadas como modalidade de ensino, pode tornálo pouco atrativo, não eficiente e desgastante para os estudantes, o que impacta negativamente na prática clínica por dificultar a compreensão da relação da teoria aprendida com as vivencias almejadas. $^{2}$

Desta forma, uma variedade de inclusões, alterações e novas tendências relacionadas ao processo de ensino e aprendizagem em saúde, apontam diretamente para necessidade da adoção de estratégias que sejam inovadoras e capazes de motivar os estudantes, melhorando seus resultados de aprendizagem. ${ }^{3}$

$\mathrm{Na}$ intencionalidade de promover o desenvolvimento e retenção de conhecimento, especificamente no âmbito da enfermagem e em contraposição ao método tradicional em que o estudante possui uma postura passiva para receber o aprendizado evidenciado, geralmente na 
modalidade de palestras, a utilização de metodologia ativa de ensino propõe o movimento inverso, compreendendo o sujeito como um agente capaz de obter um papel ativo em sua aprendizagem, posto que têm suas experiências, saberes e opiniões valorizadas como ponto de partida para construção do conhecimento..$^{4-5}$

Estratégias ativas de ensino como a elaboração de paródias sobre os temas propostos, debate entre grupos para reflexão, jogo da memória e estudo de caso, favorecem e aperfeiçoam o pensamento crítico do estudante em enfermagem, retirando-o da escuta passiva e exclusiva dos conteúdos. ${ }^{4}$ Salienta-se, desta forma, a importância de articular estratégias tradicionais e ativas de ensino e aprendizagem em enfermagem, visando o desenvolvimento de conhecimento, principalmente, diante da necessidade do ensino de temáticas mais complexas como a assistência de enfermagem ao paciente séptico. ${ }^{6-7}$

Nos últimos 30 anos, a incidência mundial de sepse aumentou cerca de $13,7 \%$ ao ano, estimando-se que, a cada ano, mais de 18 milhões de pessoas são acometidas pela síndrome e mais de 5 milhões evoluem à óbito. ${ }^{6-7}$ Estimativas apontam a existência de, aproximadamente, 600 mil novos casos de sepse a cada ano no Brasil, ocasionando um impacto direto nos indicadores de morbimortalidade do país, ao representar $16,5 \%$ dos atestados de óbitos emitidos. ${ }^{8}$

Quando um paciente inicia um quadro de sepse definida por um conjunto de manifestações graves em todo o organismo, tais como hipertermia, anorexia, mialgia, taquicardia, taquipneia, hipotensão, oligúria, irritabilidade e letargia, produzidas por uma infecção, um dos maiores desafios para os enfermagem é a incipiência de conhecimento sobre a enfermidade e a dificuldade para identificar os sinais e sintomas precocemente. ${ }^{9}$ A adoção de estratégias educacionais ativas de ensino para aprendizagem da sepse, com abordagem utilizando mecanismos pedagógicos inovadores e atrativos, pode favorecer o desenvolvimento de conhecimento desta temática e propiciar a identificação precoce dos seus sinais e sintomas e a diferenciação das fases evolutivas pela enfermagem, melhorando o prognóstico dos pacientes. ${ }^{10-11}$ 
Mesmo assim, ainda evidencia-se no panorama educacional em enfermagem um desafio para promover processos de ensino e aprendizagem eficazes e motivadores para o desenvolvimento da habilidade cognitiva dos estudantes quanto a sepse, e uma incipiência de estudos científicos que abordem a articulação de estratégias de ensino, tradicionais e ativas para este fim, o que torna relevante o aprofundamento desta temática. ${ }^{11}$

Deve-se considerar o ensino sobre a sepse na perspectiva da formação de estudantes em enfermagem e promover a articulação de variadas estratégias de ensino que possibilitem a reflexão, e o desenvolvimento deste conhecimento. ${ }^{8}$ Diante do exposto questiona-se: a articulação de estratégias tradicionais e ativas de ensino sobre sepse é capaz de aperfeiçoar o desenvolvimento de conhecimento em estudantes de enfermagem? Este estudo teve como objetivo verificar a efetividade da articulação de métodos tradicionais e ativos de ensino para desenvolver conhecimento de estudantes de enfermagem sobre sepse

\section{Método}

Estudo quase experimental, transversal, do tipo antes e depois, realizado em uma em uma universidade do interior de Minas Gerais, especificamente em um curso de graduação em enfermagem.

Foram oferecidas 30 vagas para realizar um workshop sobre sepse, voltado a estudantes de enfermagem. Este número de vagas se justificou por atender a demanda de estudantes do período de graduação especificado para esta pesquisa. Obteve-se, desta forma, 27 participantes, o que caracterizou uma amostra de conveniência.

Os critérios de inclusão foram: estudantes de enfermagem matriculados no $4^{\circ}$ semestre do curso de graduação, maiores ou igual a 18 anos e que estivessem presentes em todas as fases do workshop proposto. Esta etapa da graduação foi determinada por já possuírem 
5 | Silva DO, Silva IKT, Palmieri LS, Pires FC, Nascimento

conhecimentos sobre o funcionamento do organismo humano e o processo saúde-doença e não terem ainda contato com pacientes críticos.

A vivencia clínica em enfermarias, unidades de pronto atendimento e unidades críticas, cursos de atualização sobre a temática ou outra formação profissional na área da saúde, foram caracterizados como critérios de exclusão.

A coleta de dados foi realizada por meio de um workshop denominado: Sepse: as horas de ouro, de agosto a dezembro de 2017. A principal intencionalidade era avaliar o desenvolvimento de conhecimento sobre sepse dos estudantes de enfermagem, antes e depois da aplicação de estratégias tradicionais e ativas de ensino, imediatamente após a intervenção e depois de três meses.

Para este fim, utilizou-se um instrumento de avaliação cognitiva do tipo pré e pós-teste, elaborado pelos pesquisadores deste estudo e validado em face e conteúdo por um comitê de cinco juízes, especialistas no tema e doutores na área, selecionados pela plataforma curricular Lattes.

Os critérios definidos para avaliação dos juízes correspondiam a uma gradação numérica de 1 a 3 representados por: insatisfatório; satisfatório e excelente, respectivamente, assinaladas diante de um formulário de avaliação elaborado com o auxílio da ferramenta Google docs. Para os tópicos avaliados como insatisfatório, orientou-se justificar descritivamente a decisão.

Foram, desta forma, avaliados 7 principais tópicos, à saber: relevância do tema; profundidade da abordagem; clareza; pertinência do vocabulário; atualização e precisão dos conceitos; atualização e qualidade das referências; coerência. Destes 5 foram considerados excelentes: relevância do tema; profundidade da abordagem; atualização e precisão dos conceitos; atualização e qualidade das referências e coerência, um tópico foi considerado satisfatórios: clareza e um insatisfatório: pertinência do vocabulário. A clareza e pertinência do 
vocabulário foram avaliados pelos pesquisadores e estruturados, atendendo a justificativa dos juízes para adequação e qualidade do instrumento proposto.

O questionário de estrutura autoaplicável, embasado cientificamente nos referenciais teóricos promovidos pelo Instituto Latino Americano para Sepse, ${ }^{12}$ contém 6 questões fechadas. A primeira questão abordava as fases evolutivas da sepse, a segunda a caracterização da presença de infecção sem disfunção, a terceira definia a sepse, o choque séptico era definido na quarta questão, a quinta e sexta questão abordavam as estratégias de cuidado que compõem o pacote de tratamento de três e seis horas para sepse, respectivamente. Cada questão possui 6 alternativas, sendo apenas uma correta. A pontuação foi compreendida de 0 a 6 pontos, e o escore médio para considerar o conhecimento sobre sepse suficiente, na presente pesquisa, igual ou maior que 4,0 pontos.

Durante o preenchimento pelos estudantes, os pesquisadores permaneceram a uma proximidade física que garantiu que eles não consultassem nenhuma literatura, minimizando os riscos de esclarecimento de dúvidas ou troca de informações entre si para evitar viés de pesquisa. Ocorreram três etapas, descritas a seguir, para a coleta de dados.

A primeira etapa abrangeu o convite e inscrição dos participantes por correio eletrônico, a recepção dos mesmos no local do evento, explicação sobre a proposta e protocolo de pesquisa e aceite de participação por meio da assinatura do Termo de Consentimento Livre e Esclarecido. Após, os estudantes preencheram o instrumento de caracterização sociodemográfica e realizaram a primeira etapa do teste cognitivo para avaliar o conhecimento prévio quanto a sepse, considerado pré-teste.

Foram selecionadas, embasando-se nas diretrizes para sepse do Instituto Latino Americano de Sepse, ${ }^{12}$ as seguintes estratégias de ensino e aprendizagem: a palestra expositiva, considerada neste estudo, a estratégia tradicional de ensino, associada a elaboração e 
apresentação de paródias musicais sobre a sepse, debate em grupo, jogo da memória em equipe e estudo de caso, apontadas como estratégias ativas de ensino.

$\mathrm{Na}$ segunda etapa, todas as estratégias foram aplicadas por um profissional enfermeiro, docente em enfermagem, especialista em assistência de enfermagem, voltada a pacientes gravemente enfermos, com experiencia na utilização de métodos ativos de ensino para enfermagem.

Inicialmente foi proferida a palestra aos participantes, abordando o conceito de sepse e a caracterização de suas fases evolutivas. Em seguida, os estudantes participaram de uma oficina para elaborar e apresentar parodias educativas sobre sepse, embasados nos conceitos adquiridos na palestra. Após, houve a aplicação do debate entre equipes sobre os conceitos e características de cada fase da sepse.

A segunda etapa abordou a assistência de enfermagem pertinente ao paciente séptico utilizando a modalidade da palestra e, em seguida, a aplicação do jogo da memória sobre a assistência ao paciente em sepse realizado em grupo e a resolução de um estudo de caso referente a um paciente séptico.

O estudo de caso descreveu a história clínica de um paciente com diagnóstico médico de sepse, internado em uma enfermaria de um hospital, baseando-se nas diretrizes do Instituto Latino Americano de Sepse, ${ }^{12}$ como referencial teórico. Os participantes eram responsáveis por identificar a fase evolutiva e os cuidados de enfermagem necessários, para posterior discussão em grupo e esclarecimento de dúvidas. Após, foi realizado a avaliação do conhecimento sobre sepse com aplicação de pós-teste.

A terceira etapa da coleta ocorreu após três meses da intervenção educativa proposta. Estabeleceu-se um encontro presencial, com o comparecimento dos 27 estudantes que participaram das etapas anteriormente descritas, e aplicada a avaliação de conhecimento cognitivo sobre sepse, com a intencionalidade de avaliar a retenção da aprendizagem sobre a temática. 
Os dados coletados foram tabulados em planilhas no Programa Microsoft Excel, e validados por dupla digitação. Posteriormente, foram exportados e processados no programa Statistical PacKage for the Social Sciences (SPSS) versão 22 para Windows para análise. As variáveis qualitativas foram analisadas segundo estatística descritiva, por meio de distribuição de frequência e porcentagens, isto é; para cada item de pergunta do questionário foi levantada e obtida a frequência de cada resposta e sua respectiva porcentagem. Para a comparação dos resultados de antes e depois da articulação das estratégias tradicionais e ativas de ensino e aprendizagem sobre sepse, utilizou-se o teste de ANOVA com medidas repetidas. O nível de significância será fixado em $\mathrm{p}<0,05$.

Quanto ao cumprimento dos preceitos éticos, a pesquisa foi encaminhada para o comitê de ética a fim de se cumprir as exigências da Resolução 466/12 do Conselho Nacional de Saúde, que regulamenta as normas para a realização de pesquisas que envolvem seres humanos, obtendo parecer de aprovação número: 2.096 .720 em 02 de junho de 2017. Para participação no estudo, os estudantes foram consultados quanto ao interesse e disponibilidade e assinaram o Termo de Consentimento Livre e Esclarecido.

\section{Resultados}

Dos 27 participantes, a maioria foi representada pelo sexo feminino (92,6\%), e a faixa etária prevalente foi entre 18 a 22 anos (44,4\%), com média de idade de 20,70 e desvio padrão de 7,467. Objetivando-se avaliar o conhecimento sobre sepse dos estudantes de enfermagem, antes e após a articulação de estratégias tradicionais e ativas de ensino, aplicou-se o instrumento de avaliação cognitiva. Apresenta-se, a seguir, inicialmente, no quadro 1, o número e percentual de estudantes em enfermagem que responderam corretamente cada questão do instrumento proposto, durante o pré e pós-teste, imediatamente após a intervenção educativa e depois de três meses. 
Quadro 1. Identificação do número, percentual e média de estudantes que responderam corretamente as questões referentes ao instrumento de avaliação cognitiva sobre sepse, durante o pré e pós-teste imediato e após 3 meses. Uberaba, 2018. $(\mathrm{N}=27)$

\begin{tabular}{|l|c|c|c|c|c|c|c|}
\hline \multicolumn{2}{|c|}{ Questões } & \multicolumn{2}{c|}{ Pré-teste } & \multicolumn{2}{c|}{$\begin{array}{c}\text { Pós-teste imediato a } \\
\text { intervenço }\end{array}$} & $\begin{array}{c}\text { Pós-teste depois de } \\
3 \text { meses }\end{array}$ & $\begin{array}{c}\text { Média de } \\
\text { estudantes }\end{array}$ \\
\hline \multicolumn{1}{|c|}{ 1: Fases } & 11 & $40,74 \%$ & 22 & $81,48 \%$ & 20 & $74,07 \%$ & 17,66 \\
\hline $\begin{array}{l}\text { Questão } \\
\text { evolutivas da sepse }\end{array}$ & N & $\%$ & N & & & & \\
\hline $\begin{array}{l}\text { Questão 2: Presença de } \\
\text { infecção sem disfunção, }\end{array}$ & 8 & $29,63 \%$ & 15 & $55,55 \%$ & 13 & $48,14 \%$ & 12,00 \\
\hline $\begin{array}{l}\text { Questão 3: Definição de } \\
\text { sepse }\end{array}$ & 9 & $33,33 \%$ & 9 & $33,33 \%$ & 8 & $29,62 \%$ & 8,66 \\
\hline $\begin{array}{l}\text { Questão 4: Definição de } \\
\text { choque séptico }\end{array}$ & 11 & $40,74 \%$ & 14 & $51,85 \%$ & 14 & $51,85 \%$ & 13,00 \\
\hline $\begin{array}{l}\text { Questão 5: Estratégias } \\
\text { que compõem o pacote de } \\
\text { tratamento de 3 horas }\end{array}$ & 7 & $25,92 \%$ & 21 & $77,77 \%$ & 19 & $70,37 \%$ & 15,66 \\
\hline $\begin{array}{l}\text { Questão 6: Estratégias } \\
\text { que compõem o pacote de } \\
\text { tratamento de 6 horas }\end{array}$ & 7 & $25,92 \%$ & 20 & $74,07 \%$ & 17 & $63,00 \%$ & 14,66 \\
\hline
\end{tabular}

Evidenciou-se, ao analisar-se o número de estudantes em enfermagem que responderam corretamente cada questão pertencente ao instrumento de avaliação do cognitiva sobre sepse, que houve melhora do conhecimento, ao comparar-se os resultados de pré e pós-teste imediato a intervenção, principalmente nas questões que abordavam as fases evolutivas da sepse, com média 17,66 e as estratégias de cuidado que compõem o pacote de tratamento de 3 e 6 horas para o paciente séptico, com médias 15,66 e 14,66, respectivamente, Após 3 meses, apesar de notar-se queda nos escores de pós-teste, os resultados foram aproximados da avaliação imediata a intervenção, o que pode indicar o desenvolvimento do conhecimento e a potencialidade em articular-se estratégias ativas e tradicionais de ensino.

A seguir, a tabela 1, evidencia a comparação entre as médias do conhecimento sobre sepse dos estudantes de enfermagem por meio da aplicação do teste ANOVA para medidas repetidas. Para isso, identificou-se os escores obtidos no pré e pós-teste, imediatamente após a intervenção e depois de três meses. 
Tabela 1. Comparação entre as médias de conhecimento dos estudantes de enfermagem sobre a sepse, obtidos no pré e pós-teste, imediatamente após a intervenção e depois de três meses, Uberaba, 2018. (N=27)

\begin{tabular}{llcc}
\hline Variáveis & Média & Desvio Padrão & $\mathrm{p}$ \\
\hline Pré-teste & 1,96 & 1,93 & \\
Pós-teste imediato & 4,44 & 1,15 & $<0,001$ \\
Pós-teste - 3 meses & 4,33 & 0,92 &
\end{tabular}

Em relação à comparação entre as médias de conhecimento dos estudantes de enfermagem sobre a sepse obtidos no pré e pós-teste imediatamente após a intervenção e depois de três meses, realizada por meio do teste ANOVA para medidas repetidas, evidenciou-se um aumento significativo da média de acertos do pós-teste imediato e pós-teste após 3 meses da intervenção, quando comparado com os resultados do pré-teste. Constatou-se significância estatística com $p<0,001$.

Apesar da evidência de resultado estatisticamente significativo para o desenvolvimento de conhecimento dos estudantes de enfermagem, frente a articulação das estratégias ativas e tradicionais de ensino e aprendizagem, faz-se necessário considerar que os referidos alunos não possuíam ainda, contato com a temática da sepse, visto os critérios de inclusão definidos para o presente estudo, e com isso esperava-se constatar que os mesmos obtivessem um maior número de acertos durante o pós-teste, imediatamente após as estratégias de ensino aplicadas.

O gráfico do tipo Boxplot representado na figura a seguir, permite analisar e comparar a variação do conhecimento nos diferentes momentos de avaliação por meio de quartis, indicando o grau de dispersão, a obliquidade nos dados e os outliers.

Figura 1. Comparação da variação do conhecimento no pré-teste, pós-teste imediato e pós-teste depois de 3 meses da intervenção proposta. Uberaba, 2018. $(\mathrm{N}=27)$ 


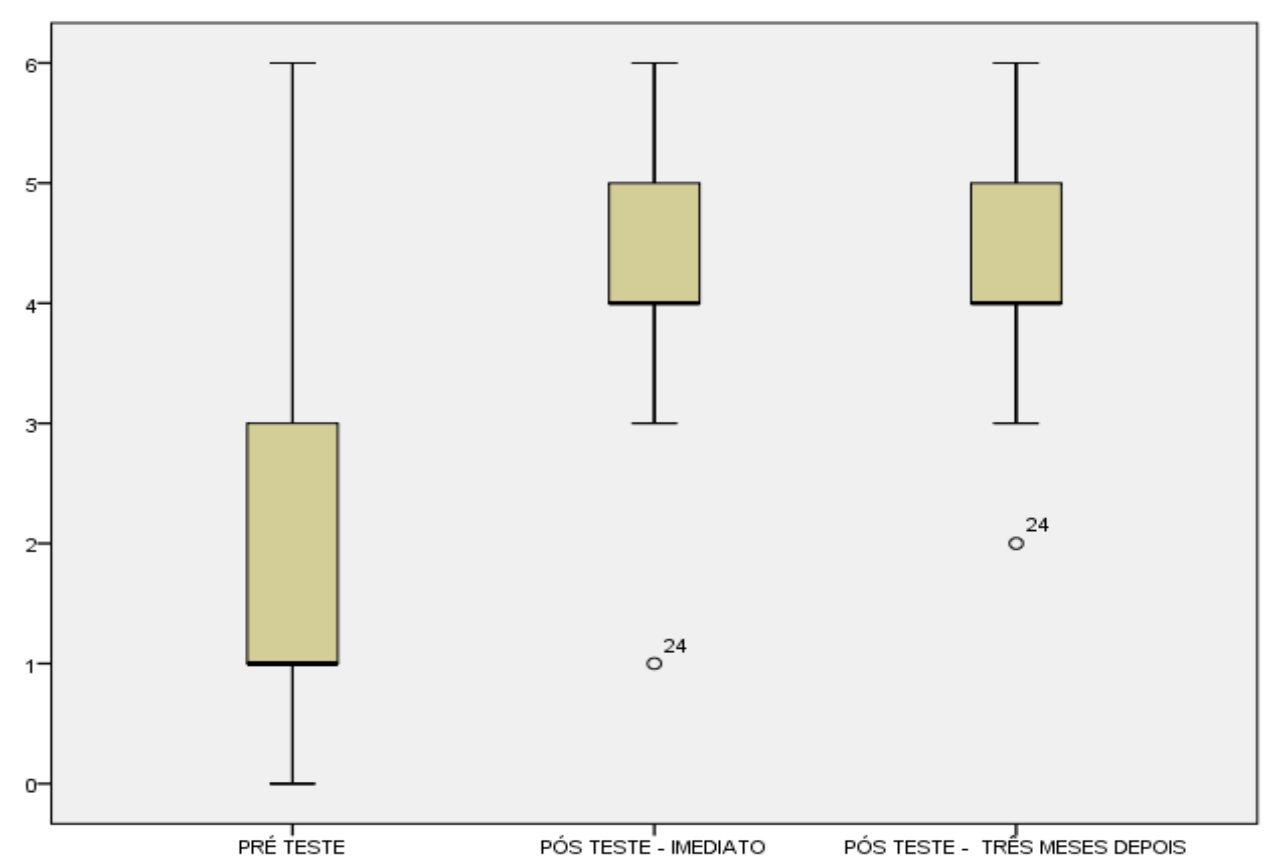

Evidenciou-se uma dispersão significativa do número de acertos referentes ao pré-teste em relação ao desempenho identificado no pós-teste imediato a intervenção e depois de três meses. Identificou-se também, a presença de apenas um participante discrepante dos demais, em ambos os momentos de pós-teste, quanto ao desenvolvimento de conhecimento cognitivo, apresentando o menor número de acertos sobre a sepse.

\section{Discussão}

O predomínio de estudantes do sexo feminino identificado neste estudo, corrobora com o cenário atual da enfermagem que aponta a figura feminina como dominante entre os trabalhadores desta área no mundo e no Brasil. ${ }^{13-15}$ Isso configura um delineamento histórico, caracterizado pelas enfermeiras que ministravam os cuidados maternos, de enfermos nos lares, de sujeitos em situação de risco ou vulnerabilidade. ${ }^{14}$ Apesar do número de mulheres ainda ser bem maior, nota-se um aumento do número de acadêmicos do sexo masculino nos cursos de graduação em enfermagem, o que torna possível afirmar que surge uma nova tendência nesse campo, o caminhar para a igualdade de gênero na categoria. ${ }^{15}$ 
Um perfil jovem dos estudantes, também foi identificado neste estudo na faixa etária entre 18 a 22 anos. Confirmando este cenário, estudo descritivo, transversal, desenvolvido em abril de 2011 a março de 2012, em quatro cursos de graduação em enfermagem brasileiros, abordando 705 acadêmicos, destacou um perfil jovem dos discentes, na faixa etária entre 20 a 24 $\operatorname{anos}(50 \%)$, com idade média de 24,21 anos. ${ }^{14}$

A presença de jovens acadêmicos nos cursos de enfermagem pode estar relacionada com o incentivo do governo brasileiro ao ingresso no ensino superior. Porém, por ser uma população jovem, a escolha da profissão pode ser imatura e ocasionar um maior índice de desistência no curso. ${ }^{14}$

Quanto a avaliação do desenvolvimento cognitiva sobre sepse, notou-se melhora do conhecimento, com ênfase em temas como as fases evolutivas da sepse e as estratégias de cuidado que compõem o pacote de tratamento de 3 e 6 horas para o paciente séptico.

Atualmente, são poucos os estudos que exploram o ensino da sepse na graduação em enfermagem, ${ }^{10-11}$ o que evidencia a necessidade de aprofundamento sobre esta questão, visto a importância do tema para o processo de ensino e aprendizagem nesta profissão e seu papel crucial na identificação precoce dos sinais e sintomas da doença. ${ }^{16}$

Pesquisas que utilizaram metodologia ativas como no presente estudo, com enfoque no ensino da sepse, também concluíram que é pertinente aos docentes em enfermagem e instituições, o investimento na articulação de estratégias educacionais, com ênfase em métodos ativos, visto seu impacto positivo no desenvolvimento de conhecimento de estudante foi favorecido por esta associação. ${ }^{10-11}$

$\mathrm{Na}$ presente pesquisa, evidenciou-se em geral, resultados estatisticamente significativos, imediatamente após a intervenção educacional aplicada. Isso pode subsidiar que, associar estratégias tradicionais e ativas de ensino é um método pedagógico eficaz para a aprendizagem da sepse. Desta forma, a utilização exclusiva da palestra expositiva pode não valorizar um espaço 
de participação para que alunos exponham suas dúvidas, reflexões e argumentações de maneira significativa, dificultando o desenvolvimento de conhecimento sobre a sepse. ${ }^{17}$

Questionamentos direcionam-se à esta estratégia por ser vista apenas como um método de transmissão de conhecimentos e por não estimular o raciocínio por parte do aluno, mas apenas a aprendizagem do tipo receptiva. ${ }^{11}$ Assim, o desenvolvimento de raciocínio clínico para uma prática está intrinsecamente relacionado ao uso de diferentes estratégias de ensino. ${ }^{18}$

Partindo do exposto, a presente pesquisa, enfatizou a associação da palestra, como estratégia tradicional de ensino com variadas estratégias que valorizam a participação dos estudantes, entre elas a elaboração e apresentação de parodias com o tema da sepse.

Um estudo realizado com 44 alunos do ensino fundamental de duas escolas municipais de Pelotas, no Rio Grande do Sul, evidenciou que após a atividade com a utilização da paródia, 100\% dos estudantes obtiveram êxito na aprendizagem proposta. ${ }^{19}$ Este resultado corrobora com a presente pesquisa, por ter adotado a elaboração de uma paródia educativa sobre a sepse e sua apresentação, o que caracterizou-se numa estratégia eficiente, de fácil utilização e divertida para o aprendizado. ${ }^{19}$

$\mathrm{Na}$ mesma linha de reflexão, o debate entre equipes favorece o diálogo, o trabalho em grupo, liderança, persuasão, de acordo com o saber criado por meio do processo coletivo. ${ }^{20-22}$

Por sua vez, o jogo da memória vem sendo apontado como uma atividade lúdica, utilizada nas salas de aulas nos últimos anos, devido a aceitação dos alunos e é capaz de garantir a construção do conhecimento por meio da interação entre os estudante, ${ }^{23}$ o que se assemelha com o jogo para memorização aplicado no presente estudo sobre sepse, e com a disponibilidade evidenciada pelos estudantes de enfermagem em sua execução.

Já, a adoção do estudo de caso foi evidenciada em um estudo realizado com estudantes de um curso de graduação em enfermagem da Universidade Federal de Viçosa, Minas Gerais. Nesta pesquisa, dividiu-se pequenos grupos sob a orientação de um tutor e aplicou-se um caso clínico, identificando satisfação dos participantes e impacto positivo quanto ao seu 
conhecimento, ${ }^{24-25}$ o que corrobora com o resultado identificado sobre o ensino da sepse, neste estudo, com a utilização de um caso clínico sobre a temática e o aperfeiçoamento cognitivo dos estudantes.

Apesar de não se ter obtido um resultado de significância estatística quanto o desenvolvimento de conhecimento dos estudantes de enfermagem após três meses da aplicação da intervenção educativa sobre sepse, cabe ressaltar que os escores identificados se assemelham com o resultado apresentado no pós-teste imediato.

As principais limitações deste estudo foram o número de participantes expostos a intervenção proposta, o que pode ter contribuído para a diminuição do poder estatístico da amostra e a incipiência de pesquisas científicas que abordam a aprendizagem da sepse em enfermagem por meio da articulação de estratégias educativas.

A contribuição deste estudo configura-se pela apresentação de uma nova perspectiva para o ensino de temáticas complexas para enfermagem como a identificação e assistência ao paciente séptico, articulando estratégias tradicionais e ativas de ensino para viabilizar a prática baseada em evidência.

\section{Conclusão}

Evidenciou-se que a articulação de métodos tradicionais e ativos de ensino e aprendizagem são capazes de provocar o desenvolvimento de conhecimento cognitivo em estudantes de enfermagem sobre sepse. Foi identificada significância estatística importante, imediatamente após a intervenção educacional aplicada, composta por palestra expositiva, paródia, debate entre equipes, jogo da memória e estudo de caso.

Após três meses da aplicação da intervenção, não se obteveresultados estatisticamente significativos para o desenvolvimento de conhecimento sobre a sepse. No entanto, cabe ressaltar que a média de acerto dos estudantes de enfermagem foi semelhante ao resultado apresentado no 
pós-teste imediato. Sugere-se, portanto, o desenvolvimento de estudos experimentais, bem delineados que se proponham a comparar a aplicação de estratégia tradicionais de ensino e estratégias ativas quanto a temática da sepse para estudantes de enfermagem.

\section{Referências}

1. Roman C, Ellwanger J, Becker GC, Silveira AD, Machado CLB, Manfroi WC. Metodologias ativas de ensino aprendizagem no processo de ensino em saúde no Brasil: uma revisão narrativa. Clin Biomed Res [Internet]. 2017 [acesso em 2018 abr 23];37(4):349-57. Disponível em: https://seer.ufrgs.br/hcpa/article/view/73911/pdf

2. Dolničar D, Podgornik BB, Bartol T. A comparative study of three teaching methods on student information literacy in stand-alone credit-bearing university courses. J Inf Scienc 2016 [Internet]. 2018 [acesso em 2018 abr 23];43(5):601-14. Disponível em: https://journals.sagepub.com/doi/10.1177/0165551516655084

3. Chamberlain J. Prebriefing in nursing simulation: a concept analysis using rodger's methodology. Clin Simul Nurs [Internet]. 2015 [acesso em 2019 abr 23];11:318-22. Disponível em: https://www.nursingsimulation.org/article/S1876-1399(15)00042-0/pdf

4. Cogo ALP, Lopes EFS, Perdomini FRI, Flores GE, Santos MRR. Construção e desenvolvimento de cenários de simulação realística sobre a administração segura de medicamentos. Rev Gaúch Enferm [Internet]. 2019 [acesso em 2019 abr 23];40:1-5. Disponível em: http://www.scielo.br/pdf/rgenf/v40nspe/1983-1447-rgenf-40-spe-e20180175.pdf

5. Diesel A, Baldez ALS, Martins SN. Os princípios das metodologias ativas de ensino: uma abordagem teórica. Rev Thema [Internet]. 2017 [acesso em 2018 abr 23];14(1):268-88. Disponível em: http://periodicos.ifsul.edu.br/index.php/thema/article/view/404/295

6. Rodrigues CCFM, Carvalho DPSRP, Salvador PTCO, Medeiros SM, Menezes RMP, Ferreira Júnior MA, et al. Ensino inovador de enfermagem a partir da perspectiva das epistemologias do Sul. Esc Anna Nery Rev Enferm [Internet]. 2016 [acesso em 2018 abr 23];20(2):384-9. Disponível em: http://www.scielo.br/scielo.php?pid=S1414-81452016000200384\&script=sci_abstract\&tlng=pt doi: 10.5935/1414-8145.20160053

7. Waltz CF, Jenkins LS, Han N. The use and effectiveness of active learning methods in nursing and health professions education: a literature review. Nurs Educ Perspect [Internet]. 2014 [acesso em $2018 \mathrm{abr}$ 23];35(6):392-400. Disponível em: https://insights.ovid.com/crossref?an=00024776-201411000-00008 doi: $10.5480 / 13-1168$ 
8. Ruiz GO, Castell CD. Epidemiologia das infecções graves nas unidades de terapia intensiva latinoamericanas. Rev Bras Ter Intensiva [Internet]. 2016 [acesso em 2017 jan 16];28(3):261-3. Disponível em: http://www.scielo.br/pdf/rbti/v28n3/0103-507X-rbti-28-03-0261.pdf doi: 10.5935/0103-507X.20160051

9. Viana RAPP, Machado FR, Souza JLA. Sepse: um problema de saúde pública: a atuação e colaboração da enfermagem na rápida identificação e tratamento da doença [Internet]. São Paulo: Conselho Regional de Enfermagem; 2017 [acesso em 2017 jan 05]. Disponível em: https://www.ilas.org.br/assets/arquivos/ferramentas/livro-sepse-um-problema-de-saude-publica-coren-ilas.pdf

10. Santos LNC, Jesus RF, Guerra KV, Santos VN. Ensino baseado em simulação: desenvolvimento do pacote de 6 horas da sepse. Rev Rede Cuid Saúde [Internet]. 2016 [acesso em 2017 jan 16];10(1):1-4. Disponível em: http://publicacoes.unigranrio.edu.br/index.php/rcs/article/view/2768

11. Parulla CD, Fragoso AS, Caregnato RCA, Canabarro ST, Flores CD. Sepse: desenvolvimento e avaliação de um curso em EAD para formação interdisciplinar. Rev Epidemiol Controle Infecç [Internet]. 2016 [acesso em 2017 jan 16];6(1):06-11. Disponível em: http://online.unisc.br/seer/index.php/epidemiologia/article/view/6334

12. Instituto Latino Americano de Sepse (ILAS). Sobre o ILAS [Internet]. 2016 [acesso em 2017 jan 16]. Disponível em: http://www.ilas.org.br/nossa-historia.php

13. Ramalho Neto JM, Campos DA, Marques LBA, Ramalho CROC, Nóbrega MML. Concepções de enfermeiros que atuam em unidade de terapia intensiva geral sobre sepse. Cogitare Enferm [Internet]. 2015 [acesso em 2017 jan 11];20(4):711-6. Disponível em: https://revistas.ufpr.br/cogitare/article/view/41963/26636

14. Bublitz S, Guido LA, Kirchhof RS, Neves ET, Lopes LFD. Perfil sociodemográfico e acadêmico de discentes de enfermagem de quatro instituições brasileiras. Rev Gaúch Enferm [Internet]. 2015 [acesso em 2017 jan 11];36(1):77-83. Disponível em: https://seer.ufrgs.br/RevistaGauchadeEnfermagem/article/view/48836

15. Machado $\mathrm{MH}$, Wermelinger M, Vieira M, Oliveira E, Lemos W, Aguiar Filho W, et al. Aspectos gerais da formação da enfermagem: o perfil da formação dos enfermeiros, técnicos e auxiliares. Enferm Foco (Brasília) [Internet]. 2016 [acesso em 2017 jan 15];6(2/4):15-34. Disponível em: http://revista.cofen.gov.br/index.php/enfermagem/article/viewFile/687/297

16. Silva RC, Cazella SC, Caregnato RCA. Metodologia da problematização na educação à distância sobre sepse: um curso com o apoio do Arco de Maguerez. Rev Latinoam Tecnol Educ [Internet]. 2018 [acesso em 2018 jul 24];17(1):121-34. Disponível em: https://relatec.unex.es/article/view/3227/2197

17. Carter AG, Creedy DK, Sidebotham M. Efficacy of teaching methods used to develop critical thinking in nursing and midwifery undergraduate students: a systematic review of the literature. Nurse Educ Today. 2016;40:209-18. 
18. Mesquita AR, Souza WM, Boaventura TC, Barros IM, Antoniolli AR, Silva WB, et al. The effect of active learning methodologies on the teaching of pharmaceutical care in a brazilian pharmacy faculty. PLos ONE. 2015;10(5).

19. Velasco RS, Rostas MHSG, Nascimento CT, Barros G. O ensino de língua materna através de paródias como ferramenta educacional. Rev Thema [Internet]. 2017 [acesso em 2018 jul 24];14(3):174-83. Disponível em: http://periodicos.ifsul.edu.br/index.php/thema/article/view/575/690

20. Montoya MAM. Multidisciplinary approach of a didactic strategy for the second year of high school. J Chem Chem Eng. 12(2018);83-6. Disponível em: http://www.davidpublisher.org/Public/uploads/Contribute/5b6a66c0650b3.pdf

21. Carter AG, Creedy DK, Sidebotham M. Efficacy of teaching methods used to develop critical thinking in nursing and midwifery undergraduate students: a systematic review of the literature. Nurse Educ Today. 2016;40:209-18. Disponível em: https://www.ncbi.nlm.nih.gov/pubmed/27125175 doi: 10.1016/j.nedt.2016.03.010

22. Oliveira CL, Silva LT. Discussão e técnicas de ensino em grupo: ferramentas de aprendizagem no ensino do direito. Rev Eletrônica Direito e Política. 2015;10(4):2187-208.

23. Ricchi A, Martelli E, Molinazzi MT, Vaccari S, Messina MP, Banchelli F, et al. Survey of students of the degree course in obstetrics, on learning using case based learning (cbl) method in the area of professional teachings. Rev Bras Clín Ter. 2018 set-out;169(5):e213-e216.

24. Carbogim FC, Oliveira LB, Mendonça ET, Marques DA, Friedrich DBC, Püschel VAA. Ensino das habilidades do pensamento crítico por meio de Problem Based Learning. Texto \& Contexto Enferm [Internet]. 2017 [acesso em 2018 jul 23];26(4):e1180017. Disponível em: http://www.scielo.br/pdf/tce/v26n4/0104-0707-tce-26-04-e1180017.pdf

25. Garcia IM, Borges TAP, Pimentel RRS, Vannuchi MTO. Percepção do discente de enfermagem na construção do seu conhecimento no contexto da metodologia ativa. Rev Eletrônica Acervo Saúde. [Internet]. 2019 [acesso em 2018 jul 23];11(2):e127. Disponível em: https://acervomais.com.br/index.php/saude/article/view/127/89

\section{Autor correspondente}

Juliana da Silva Garcia Nascimento

E-mail: mestradounesp28@yahoo.com.br

Endereço: Rua Osório Joaquim Guimarães, no 281, Ap. 203, Bloco:05, Parque São Geraldo. Uberaba- Minas Gerais, Brasil. CEP: $38030-130$ 


\section{Contribuições de Autoria}

\section{1 - Douglas de Oliveira Silva}

Concepção do estudo; coleta de dados; Análise e interpretação dos dados, discussão dos resultados.

\section{2 - Izabela Katiucia Tomaz da Silva}

Concepção do estudo; coleta de dados; Análise e interpretação dos dados, discussão dos resultados.

\section{3 - Laís Silva Palmieri}

Concepção do estudo; coleta de dados; Análise e interpretação dos dados, discussão dos resultados.

\section{4 - Fabiana Cristina Pires}

Concepção do estudo; discussão dos resultados, redação e revisão crítica do conteúdo; revisão e aprovação da versão final

\section{5 - Juliana da Silva Garcia Nascimento}

Concepção do estudo; coleta de dados; Análise e interpretação dos dados, discussão dos resultados, redação e revisão crítica do conteúdo; revisão e aprovação da versão final.

\section{Como citar este artigo}

Silva DO, Silva IKT, Palmieri LS, Pires FC, Nascimento. Estratégias de ensino para a aprendizagem sobre sepse. UFSM. 2020 [Acesso em: Anos Mês Dia]; vol.10 e17: 1-.18 DOI: https://doi.org/10.5902/2179769236364 